\title{
Recent Techniques for Harvesting Energy from the Human Body
}

\author{
Nidal M. Turab ${ }^{1}$, Hamza Abu Owida ${ }^{2}$, Jamal I. Al-Nabulsi ${ }^{2, *}$ and Mwaffaq Abu-Alhaija ${ }^{1}$ \\ ${ }^{1}$ Department of Networks and Information Security, Faculty of Information Technology, Al-Ahliyya Amman University, Amman, \\ 19328, Jordan \\ ${ }^{2}$ Medical Engineering Department, Faculty of Engineering, Al-Ahliyya Amman University, Amman, 19328, Jordan \\ ${ }^{*}$ Corresponding Author: Jamal I. Al-Nabulsi. Email: j.nabulsi@ammanu.edu.jo \\ Received: 19 February 2021; Accepted: 27 April 2021
}

\begin{abstract}
The human body contains a near-infinite supply of energy in chemical, thermal, and mechanical forms. However, the majority of implantable and wearable devices are still operated by batteries, whose insufficient capacity and large size limit their lifespan and increase the risk of hazardous material leakage. Such energy can be used to exceed the battery power limits of implantable and wearable devices. Moreover, novel materials and fabrication methods can be used to create various medical therapies and life-enhancing technologies. This review paper focuses on energy-harvesting technologies used in medical and health applications, primarily power collectors from the human body. Current approaches to energy harvesting from the bodies of living subjects for self-powered electronics are summarized. Using the human body as an energy source encompasses numerous topics: thermoelectric generators, power harvesting by kinetic energy, cardiovascular energy harvesting, and blood pressure. The review considers various perspectives on future research, which can provide a new forum for advancing new technologies for the diagnosis, treatment, and prevention of diseases by integrating different energy harvesters with advanced electronics.
\end{abstract}

Keywords: Harvesters; implantable medical devices; thermoelectric generators; cardiovascular systems; human motion harvesters

\section{Introduction}

The latest innovations in wearable and implantable devices have drawn considerable academic and industrial interest because of their prominent use in care management, disease prevention applications, treatment, and diagnosis [1-3]. Most applications are still powered by batteries, but small battery capacity and large battery sizes pose obstacles. Moreover, a higher battery capacity is undesirable because battery capacity and battery volume are usually related linearly [4,5].

Up-and-coming technologies have been created to derive energy from the natural environment by using energy harvesters for power generation. Energy harvesters can supplement battery lifespan or act as a single power supply by extending the battery life of wearable and implantable devices $[4,5]$.

This work is licensed under a Creative Commons Attribution 4.0 International License, which permits unrestricted use, distribution, and reproduction in any medium, provided the original work is properly cited. 
While many energy sources are relevant to harvesters, including sunlight, environmental IR light, and inductive coupling for radio-frequency energy [6], living entities are particularly favorable sources of energy given their large number and the diversity of available sources of energy [7,8]. For example, theoretical calculations showed that heating, breathing, and walking could produce $2.8-4.8 \mathrm{~W}, 0.83 \mathrm{~W}$, and $60 \mathrm{~W}$, respectively [7]. Although these power sources can provide excellent accommodation for the service of a pacemaker $(50 \mu \mathrm{W}$ for 7 years), a hearing aid ( $1 \mathrm{~mW}$ for 5 days), or a smartphone (1W for $5 \mathrm{~h})$ [9], proof of the viability of such electronics is needed.

Owing to the different approaches to energy harvesting from live topic electronics, this review paper specifically focuses on power energy harvesting from the human body. Examples of using the human body as an energy source include thermoelectric generators, energy harvesting using kinetic energy, and energy harvesting using the cardiovascular system and blood pressure.

\section{Energy Harvesting Using Thermoelectric Generators}

The thermoelectric generator principle refers to harvesting energy resulting from the differences between two surfaces; one is hot while the other is cold. For human body temperature, the skin is usually hot (around $32^{\circ} \mathrm{C}$ ) while the ambient temperature is cold (depending on the weather and other surroundings), as shown in Fig. 1.Thermal Energy Generators (TEGs) are solid-state devices that convert temperature differences to energy using the Seebeck effect, which can power medical devices [10]. Thus, thermal properties and thermal resistance should be considered during device optimization to attain the maximum power from wearable self-powered TEGs.

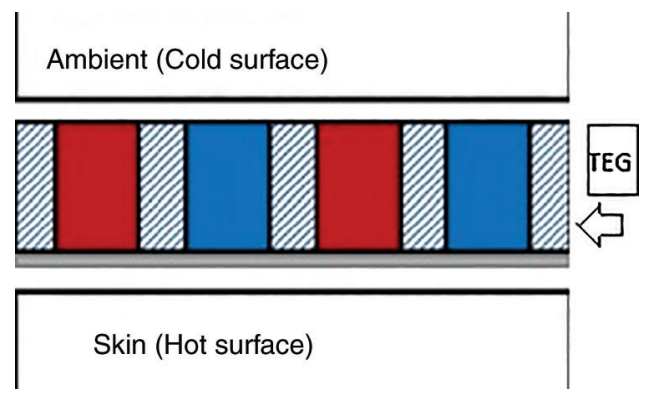

Figure 1: TEG placed between the skin and ambient surface

Reference [11] used 12 thermoelectric columns of (Bi-Sb-Te) P-type and (Bi-Se-Te) n-type materials fabricated by dispenser printing and involved experiments on different body locations, including the wrist, upper arm, chest,TEG was further fabricated into a T-shirt (as an uncontrolled hot surface) (Fig. 2). The generated power was ordered from highest to lowest value on the upper arm (power density generated $» 20 \mu \mathrm{W} / \mathrm{cm}^{2}$ ) and then the wrist (power density generated » $12 \mu \mathrm{W} / \mathrm{cm}^{2}$ ), the chest (power density generated $\gg 8 \mu \mathrm{W} / \mathrm{cm}^{2}$ ), and the T-shirt (power density generated $» 6 \mu \mathrm{W} / \mathrm{cm}^{2}$ ), with $\Delta \mathrm{T} » 7^{\circ} \mathrm{C}$ for all measurements.

Reference [12] added the four layers from the previous study for a total of 18 thermocouples of P-type and N-type thermoelectric blocks welded on a flexible printed circuit board (FPCB). The prototyped TEG produced an output power of $8.3 \mu \mathrm{W} \Delta \mathrm{T}$ of $11 \mathrm{~K}$. When the TEG was worn on the wrist, it had an output power of $130.6 \mathrm{nW}$ at $\Delta \mathrm{T}$ of $7 \mathrm{~K}$.

Reference [13] proposed thermoelectric nanocomposite materials based on microwave-based bismuth telluride alloys. The TEG produced $44 \mu \mathrm{W} / \mathrm{cm}^{2}$ under no airflow condition and $156.5 \mu \mathrm{W} / \mathrm{cm}^{2}$ under the airflow condition. 


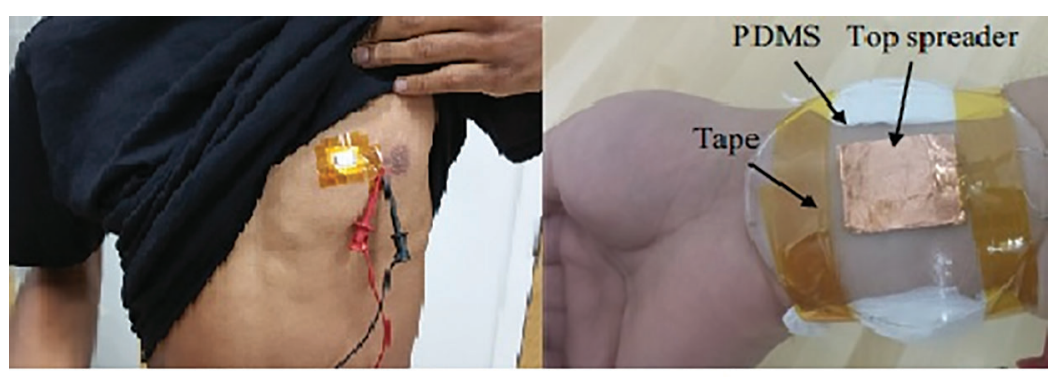

(a)

(b)

Figure 2: (a) TEG device on the skin over the chest area; (b) TEG device on the wrist

Reference [14] proposed a fabricated TEG composed of stretchable rigid P and N-type thermoelectric legs connected in a series. The authors used liquid metal as interconnections between the thermoelectric legs to provide high stretchability and low electrical resistance. When worn on the wrist, the proposed TEG produced a power density of » $35 \mu \mathrm{W} / \mathrm{cm}^{2}$ at air velocities corresponding to that of the average human walking speed.

Moreover, reference [15] proposed stretchable thermoelectric $\mathrm{P}$-and $\mathrm{N}$-type legs linked in sequence. The authors used liquid metal as interconnections between thermoelectric legs to provide a low electrical resistance stretch capacity. However, when worn on the wrist, the proposed TEG generated a power density of $» 35 \mu \mathrm{W} / \mathrm{cm}^{2}$ at air speeds corresponding to humans' average walking speed. Reference [16] proposed a prototype assembled with cellulose nanofiber and (Bi-Te) biocomposite film. The TEG prototype generated an output power of $561 \mathrm{nW}$ at an experimental $\Delta \mathrm{T}=60^{\circ} \mathrm{C}$, while the output voltage was $\sim 3 \mathrm{mV}$, with $\Delta \mathrm{T}$ of $15^{\circ} \mathrm{C}$. Reference [17] developed one-dimensional PEDOT: PSS fibers that exhibited a high tensile strength. The prototype fibrous TEG consisted of five pairs of p-type PEDOT: PSS fibers and n-type wires that delivered an output power density of $\sim 1.79 \mu \mathrm{W} / \mathrm{cm}^{2}$ at $\Delta \mathrm{T}=10^{\circ} \mathrm{C}$.

Reference [18] proposed two energy harvesters for IoT applications. The first utilizes RF signals while the second is of interest because it represents a TEG composed of two plates. A hot plate, fabricated with aluminum, and a cold plate, also manufactured with aluminum, are used as a heat diffuser, as shown in Fig. 3. The insulator is made of Teflon, which is inserted between the two cold plates. The harvested energy at $\Delta \mathrm{T}=15^{\circ} \mathrm{C}$ was $0.530 \mathrm{~J}$.

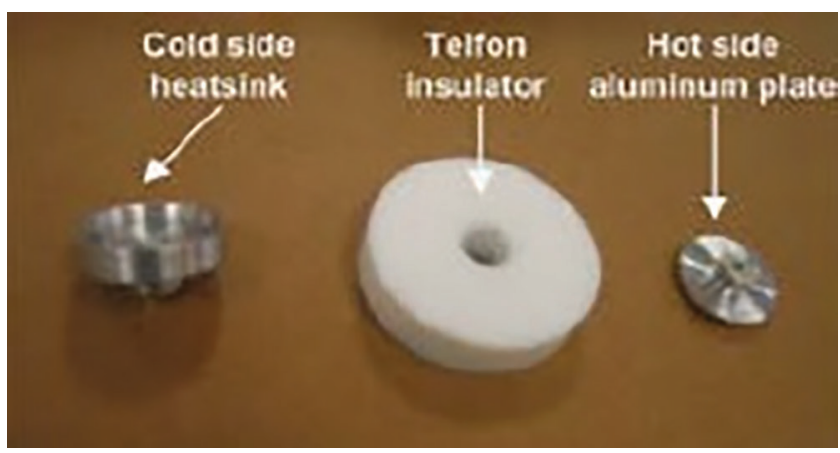

Figure 3: Two Aluminum plates TEG

Reference [19] proposed a TEG architecture composed of electrically and thermally conductive liquid metal embedded elastomer (LMEE) composites with integrated arrays of n-type and p-type Bi-Te. The TEG generated voltages of $59.96 \mathrm{mV}, 130 \mathrm{mV}$, and $278.6 \mathrm{mV}$ at $\Delta \mathrm{T}$ of $10^{\circ} \mathrm{C}, 30^{\circ} \mathrm{C}$, and $60^{\circ} \mathrm{C}$, respectively. 
Reference [20] proposed a highly efficient, flexible thermoelectric generator (f-TEG) that utilizes bismuth telluride grains assembled on flexible polyimide substrate for wrist-wearing applications. They found that the generated power density was $\gg 3.5 \mu \mathrm{W} / \mathrm{cm}^{2}$, and it reached $64.1 \mu \mathrm{W} / \mathrm{cm}^{2}$ under a wind speed of $2.5 \mathrm{~m} / \mathrm{s}$ (corresponding to average walking speed).

Reference [21] used an electrically conducting sewing thread to produce a polymer-based textile TEG. It produced a power of $1.2 \mu \mathrm{W}$ at $\Delta \mathrm{T}=65 \mathrm{~K}$ and $0.2 \mu \mathrm{W}$ at $\Delta \mathrm{T}=30 \mathrm{~K}$. Reference [22] developed a wearable double-chain thermoelectric generator (DC-TEG) to integrate thermal energy harvesting and capacitancebased multi-functional sensing. The proposed DC-TEG was fabricated using a screen-printing process to print B-Te-Se (n-type)- and Sb-Te (p-type)-based thermoelectric inks, thus forming double-chain thermocouples. The DC-TEG produced an open-circuit voltage of $151 \mathrm{mV}$ at $\Delta \mathrm{T}=50^{\circ} \mathrm{C}$, which was stored in capacitors to achieve DC voltage » $3.3 \mathrm{~V}$.

Reference [23] achieved a high thermo-generated peak power of $5 \mu \mathrm{W}$ in a gelatin-based ionicthermoelectric (i-TE) material utilizing the thermo-diffusion effect and thermo-galvanic effect (a thermogalvanic cell is a galvanic cell in which heat is employed to provide electrical power). Reference [24] integrated TEG and storage batteries. Their proposed TEG consists of 96 thermocouples that delivered a power density of up to $8.7 \mu \mathrm{W} / \mathrm{cm}^{2}$; the output voltage was around a few tens of millivolts. They used Li-S batteries to store energy and achieve an output voltage » $2.4 \mathrm{~V}$, which is suitable for most medical sensors. Reference [25] wove textile from thermoelectric fibers to produce an unobtrusive thermoelectric module. Mutually doped carbon nanotube fibers wrapped with acrylic fibers are woven into $\pi$-type thermoelectric modules. This textile generator produced an output power density of $70 \mathrm{~mW} / \mathrm{cm}^{2}$ at $\Delta \mathrm{T}=44 \mathrm{~K}$ and excellent stretchability.

Finally, reference [26] proposed a flexible thermoelectric generator (F-TEG) placed on the chest, where a polyimide (PIM) flexible substrate provides low thermal conductivity and minimizes heat loss through the n-B-Te thermoelement. The F-TEG generated a maximum output power $1.20 \mathrm{~mW} / \mathrm{cm}^{2}$ at $\Delta \mathrm{T}$ between $5 \mathrm{~K}$ and $7 \mathrm{~K}$. Reference [27] developed a flexible and wearable wristband thermoelectric consisting of seven fiber-based pairs of $\mathrm{P}-\mathrm{N}$ legs, which generated a maximum voltage of $\sim 0.18 \mathrm{mV}$ at $\Delta \mathrm{T}=10^{\circ} \mathrm{C}$. Moreover, the generated power reached up to $101.51 \mathrm{pW}$ after swinging the arm for $5 \mathrm{~min}$.

\section{Energy Harvesting from Heartbeat (Cardiovascular System):}

The human heart is an organic pump that circulates blood throughout the body via the circulatory system; a human adult heart pumps about 6,000-7,500 liters of blood daily [27]. The heart has the stamina of 42.5 billion cycles within 70 years of life; this continuous heart movement has been utilized in different ways to harvest energy. Reference [28] proposed a mass-imbalance oscillation generator consisting of a commercial automatic wristwatch (after removing all unnecessary parts to reduce weight). A clock was used as an energy-harvesting device to convert the kinetic energy from the wearer's cardiac wall motion to electrical power. The authors also developed a mathematical model to optimize the device's configuration. They conducted in vitro and in vivo experiments. In the in vitro experiment, an arm robot accelerated the clock by reproducing the cardiac motion, while in vivo experiment, the device was attached to a sheep heart for one hour. The generated power for the in vitro was $30 \mu \mathrm{W}$, and in vivo, it was $16.7 \mu \mathrm{W}$.

Reference [29] proposed linear low-frequency and nonlinear mono-stable and robust bi-stable harvesters for heart rate variation. They were designed according to the unique signature of heart vibrations. The authors investigated the bi-stable hybrid device's frequency sensitivity by examining the relationship between the optimal design's power output and the heart rate. They found that the output power of the bi-stable energy harvester was more than $3 \mu \mathrm{W}$. 
Reference [30] studied arterial expansion and contraction using electromagnetic induction. Both theoretical and experimental investigations were applied to harvest energy from the artery inserted into a laboratory-fabricated flexible coil and two arteries of a Göttinger mini pig. The average harvestable power was $42 \mathrm{nW}$.

Additionally, the authors of [31] proposed a flexible and highly efficient energy harvester where piezoelectric material (PMN-PT) was a single crystalline (1-x) $\mathrm{Pb}(\mathrm{Mg} 1 / 3 \mathrm{Nb} 2 / 3) \mathrm{O}^{3}$ xPbTiO3. They stated that the maximum output current and voltage were $145 \mu \mathrm{A}$ and $8.2 \mathrm{~V}$, respectively. Fig. $4 \mathrm{a}$ illustrates the (PMN-PT) schematic diagram and (b) the vivo experiment on the harvester on a living rat.
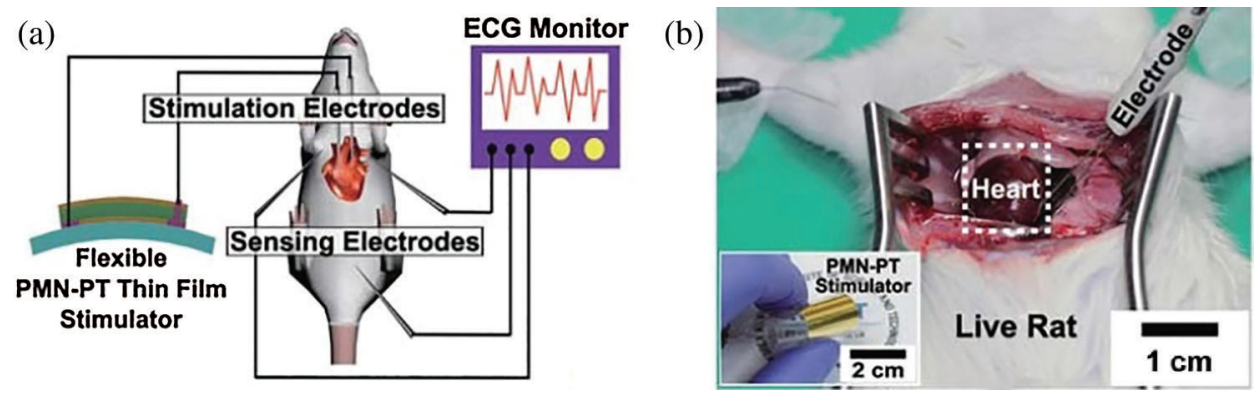

Figure 4: (a) PMN-PT energy harvester of the artificial cardiac pacemaker; (b) a vivo experiment on a living rat

Reference [32] proposed a miniaturized endocardial electromagnetic energy harvester for leadless cardiac pacemakers in vitro and in vivo tests. In this work, the authors implanted the piezoelectric generator to wrap around the ascending aorta; they found that the in vivo output and power were lower than those in vitro. The reported output power was about $30 \mathrm{nW}$. An ultra-flexible piezoelectric device based on PZT ceramics was proposed by [33], and in vivo experiments on pigs were conducted; the output voltage was about $3 \mathrm{Vp}$-p when the ultra-flexible piezoelectric device was fixed from the top of the left ventricle to the right ventricle.

Reference [34] studied an energy harvester that fan-folded a bimorph piezoelectric beam configuration with an added tip mass. The authors mentioned that high natural frequency is a significant concern in microscale energy harvesters. As this energy harvester's size is $2 \mathrm{~cm}$ by $0.5 \mathrm{~cm}$ by $1 \mathrm{~cm}$, the natural frequency is very high. They utilized the fan-folded geometry and added tip mass to reduce the natural frequency to the desired range. The reported average power was $10.24 \mu \mathrm{W}$.

A previous study applied the electromagnetic induction principle to harvest energy by using copper coils connected in a series to a permanent magnet stack suspended between two flexures [35]. A mathematical model to simulate three heart motions was developed in this work. The predicted output powers of the model were $14.5,41.9$, and $16.9 \mu \mathrm{W}$, respectively. Two in vivo experiments on domestic pigs harvested a mean output power of $0.78 \mu \mathrm{W}$ and $1.7 \mu \mathrm{W}$ at a heart rates of $84 \mathrm{bpm}$ and $160 \mathrm{bpm}$.

Reference [36] developed a low-frequency vibration energy harvester used to run implantable pacemakers and cardioverter defibrillators (AICDs). The authors used porous polyvinylidene fluoridetrifluoroethylene (PVDF-TrFE) thin film to harvest the mechanical energy from the heartbeat. The maximum electrical output was $0.5 \mathrm{~V}$ and $43 \mathrm{nA}$ under the frequency of $1 \mathrm{~Hz}$. They stated that adding a proof mass of $31.6 \mathrm{mg}$ on the dual-cantilever tip resulted in the power increasing by 1.82 times. Reference [37] tested an Implantable Piezoelectric Generator (iPEG) on a porcine heart in vivo, with a harvested maximum output power of about $33 \mu \mathrm{W}$.

Reference [38] proposed a polymer-based piezoelectric material of PVDF-TrFE that was employed to utilize the bending and twisting motion of the lead of a cardiac pacemaker. Both energy-harvesting and 
sensing devices were flawlessly combined with the existing pacemaker. The energy harvester converted energy flows connected to a lead motion into electrical power. The obtained usable capacity on the capacitor was estimated as $0.3 \mu \mathrm{W}$. A miniaturized endocardial electromagnetic energy harvester was proposed by [39] and consisted of two central units: the power unit and the oscillation unit. Both units were integrated into a tubular housing, as shown in Fig. 5. The proposed energy harvester has dimensions of $30 \mathrm{~mm} \times 7 \mathrm{~mm}$, a volume of $1.15 \mathrm{~cm}^{3}$, and a weight of only $8.01 \mathrm{~g}$. The output power was $4.2 \mu \mathrm{W}$ and $2.6 \mu \mathrm{W}$ for the bench and in vivo experiments, respectively.

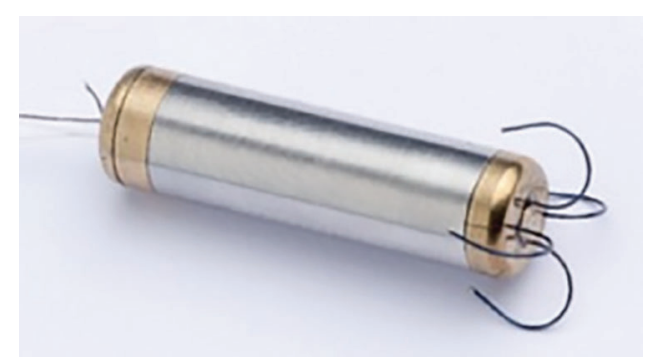

Figure 5: Miniaturized endocardial electromagnetic energy harvester

A multibeam energy harvester that consisted of $\mathrm{ZnO}$ nanoparticles $(30 \% \mathrm{wt})$ and MWCNTs $(0.1 \% \mathrm{wt})$ was proposed in [40], in which a rectifier and capacitor combined the energy harvester. They reported that the obtained energy was about $6.5 \mu \mathrm{J}$ (A $120-\mu \mathrm{F}$ capacitor charged for $34.6 \mathrm{~S}$ ), thus producing an output power of $0.187 \mu \mathrm{W}$. Reference [41] proposed another approach utilizing heart motion to harvest energy from arterial blood pressure. The proposed streaked cylinder design aims to reduce the natural frequency of the harvester by replacing the linear plate by a curved plate. The proposed harvester comprises a cylinder composed of PVDF with a conductor coated on the cylinder's inner and outer surfaces and a shunt resistor. The results showed that for the shunt resistor of $50 \Omega$, the output power was » $27.5 \mu \mathrm{W}$; the shunt resistor of $500 \Omega$ produced an output power of » $20 \mu \mathrm{W}$. Reference [42] proposed a micro-plate covered by a piezoelectric material to harvest energy from blood pressure variation. The unimorph piezoelectric energy harvester, shown in Fig. 6, created a PZT layer bonded to the substructure layer. The obtained output power was about $3.07 \mathrm{nW}$ for a plate of $84 \times 10^{-6} \mathrm{~cm}^{3}$. The output power increased with increasing pressure frequency and attained its maximum value at a load resistance of about $5 \mathrm{k} \Omega$.

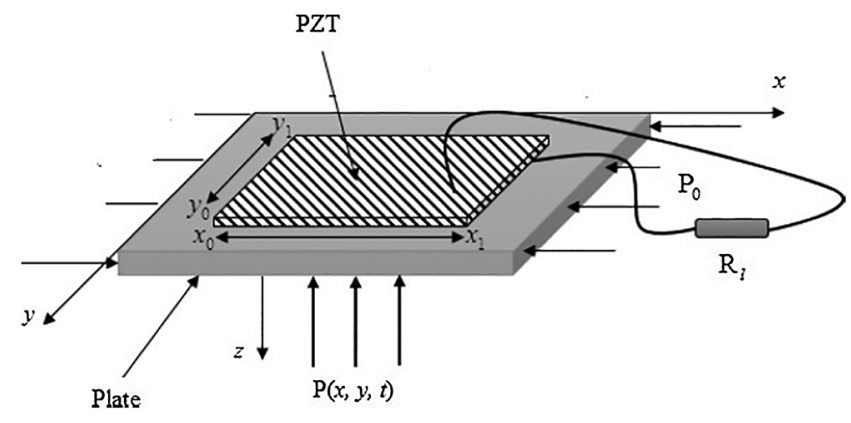

Figure 6: Unimorph piezoelectric energy harvester

Reference [43] developed an energy harvester utilizing a tunable resonance cantilever mechanism that in turn uses a single magneto-electro-mechanical tool for maximizing the energy-harvesting output by adjusting the resonant frequency (see Fig. 7). The harvested power was between $3.0 \mu \mathrm{W}$ and $20.6 \mu \mathrm{W}$ at heart rates of 79 and 243 bpm, respectively. 


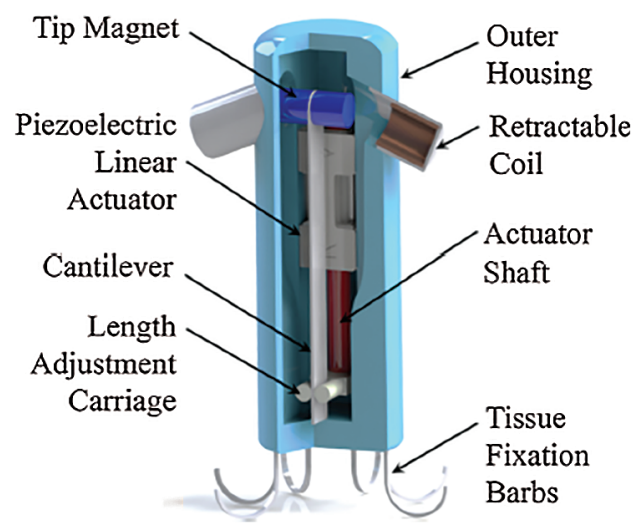

Figure 7: Tunable resonance cantilever mechanism energy harvester

Reference [44] proposed an energy-harvesting device that captures energy from arterial wall pulsation. The proposed design consists of a flexible coil that can bend along with the artery in a magnetic field by two permanent parallel ring magnets. The output power for various coils at various heart rates reached a maximum of $1.357 \mu \mathrm{W}$ at a heart rate of $235 \mathrm{bpm}$.

Reference [45] involved a study of the optimum number, dimensions, and configuration of energy harvesters attached to the pacemaker leads inside superior vena cava (SVC) to harvest energy from blood flow. The authors carried out simulation and found that a matching resistance of $35 \mathrm{M} \Omega$ produced a maximum power of $» 9 \mu \mathrm{W}$ with eight harvester beams.

\section{Energy Harvesting Using Human Motion}

Amid the recent explosive growth of wearable devices, many researchers have become drawn to harvesting electricity from human movement for medical and health needs.

Reference [46] introduced a highly effective and durable human biomechanical energy harvesting by a waterproof, intelligent insole based on a triboelectric nanogenerator. It completely utilized the footprint pressure distribution to produce power rises of $580 \mu \mathrm{W}$. Besides being able to endure extreme weather conditions, the insoles are suitable for pluvial areas, too. A total of 260 LEDS, with swarming feet and water on the field, lit-up light-emitting diodes, and a condenser of $88 \mu \mathrm{F}$, were charged at $2.5 \mathrm{~V}$ per $900 \mathrm{~s}$.

Reference [47] constructed a polyvinylene difluoride (PVDF) film-based hybrid triboelectricpiezoelectric nanogenerator (TP-NG) to effectively extract random and irregular forms of vibrator energy from human feet. The TP-NG consisted of PVDF polymer film and an Al electrode, and the acrylic support film had a stacking structure that provided power up to $127 \mu \mathrm{W}$. The toes, arch, and heel were embedded into three hybrid TP-NGs. The shoe insole produced adequate power during normal walking to operate light-emitting diodes that could be applied in night lighting. The insole operated a Wi-Fi pressurized network, enabling the pressure distribution on the foot to be tracked and transmitted to a mobile phone.

Moreover, reference [48] proposed a piezoelectric nanogenerator based on electrospun PVDF nanofibers for walking energy-harvesting, with experimental characterization and finite element analysis. An integrated PVDF nanofiber nanogenerator was simulated for use in a shoe insole as an energy harvester. The response of the nanogenerator system under a low-frequency impact was evaluated, and the maximum generated voltage was determined to be around $15.1 \mathrm{~V}$. 
Parasites of the mechanical energy in shoes originated in human motion were studied in [49]. A sandwich form with a slim thickness is readily compatible with the feet and can be used as the harvester. The high performance and outstanding reliability were also taken into account. During a walk at a frequency of about $1 \mathrm{~Hz}$, the harvester had an average power output of $1 \mathrm{~mW}$.

Reference [50] reported that piezoelectric nanogenerator was fabricated by mixing various materials such as piezoelectric $\mathrm{BaTiO} 3$ and piezoelectric $\mathrm{ZnO}$ nanoparticles with the sections of the graphene nanosheets in a silicone matrix. The findings showed a higher performance in comparison to other ceramics of PZT-based composites. Following that, a completely working nanogenerator shoe-insole was developed to demonstrate the practical application of PENGs. The nanogenerator shoe-insole generated a power density of $402 \mathrm{~mW} / \mathrm{m}^{2}$ and a highly stretchable biomechanical piezoelectric sensor under the realtime human walking method. Tab. 1 summarizes the highest power outputs obtained in the studies discussed.

Table 1: Highest power outputs given in reviewed works

\begin{tabular}{ll}
\hline Power $(\mathrm{W})$, Power density $\left(\mathrm{W} / \mathrm{m}^{2}\right)$, Voltage $(\mathrm{V})$ & Reference \\
\hline $39 \mu \mathrm{W}$ & {$[5]$} \\
$156.5 \mu \mathrm{W} / \mathrm{cm}^{2}$ & {$[8]$} \\
$151 \mathrm{mV}$ & {$[13]$} \\
$8.2 \mathrm{~V}, 145 \mu \mathrm{A}$ & {$[22]$} \\
$41.9 \mu \mathrm{W}$ & {$[26]$} \\
$15.1 \mathrm{~V}$ & {$[39]$} \\
$1 \mathrm{~mW}$ & {$[40]$} \\
$402 \mathrm{~mW} / \mathrm{m}^{2}$ & {$[41]$} \\
\hline
\end{tabular}

\section{Conclusions}

The growing prevalence of different diseases today has led to the rapid growth of Implantable Medical Devices (IMDs) powered by batteries with energy-restrictive problems. IMD battery replacement stemming from a battery's end of life requires surgical intervention for the patient, which poses risks of infection and bleeding. Also, extending battery life can lead to extending the size of an IMD such as a Pacemaker. An alternative to surgical intervention is to extend wires for charging an IMD through the human skin, but this is extremely uncomfortable and carries the risk of irritation and contamination. To overcome these limitations, a method for energy harvesting from the human body is necessary. It should allow for designing a battery-less IMD with no extra wires needed and a reduced number of surgical interventions. This article reviewed different state-of-the-art power harvesting techniques that utilize the human body, both internal and external. We concluded that the human body is a usable and renewable energy source. For each harvesting technique, various methods were presented, and the obtained results were outlined. Limitations and related options were also suggested.

The typical power consumption of a modern pacemaker is around $15 \mu \mathrm{W}$ and $40 \mu \mathrm{W}$ for defibrillators. The obtained results from the three harvesters discussed in the sections mentioned above showed that the maximum power came from human motion (about $1 \mathrm{~mW}$ ) and then from thermoelectric generators (about $41.9 \mu \mathrm{W}$ ). The last one was for the cardiovascular system (about $39 \mu \mathrm{W}$ ). The same situation was applied to the harvested output voltage and harvested output power density. The obtained power fulfilled (predominantly) the power requirements of implantable devices. 
Nevertheless, in terms of drawbacks, the cardiovascular system and other internal harvesters, such as the respiratory harvester system, suffer from problems of technological biocompatibility. All in vitro experiments were done on one animal, and no in vitro experiments were conducted on human beings to test the possible side effects of such harvesters on human health. The harvester's size is another important factor, but nanotechnology is a promising solution for minimizing harvester size.

The first issue for human motion harvesters is that they are not suitable for people with disabilities; even for non-disabled people, the harvesters' weight and size are other important concerns. The second issue concerns the transfer of harvested power to the IMD; one possible solution is to use wireless power transmission.

Wearable thermoelectric generators should be flexible, comfortable, twistable, and durable and should be worn on different parts of the body. However, the limitation of thermoelectric generators is the temperature difference between the ambient environment and the skin, which should not be extreme. A $\Delta \mathrm{T}$ value of 60 was cited in some literature, but it is not practical for most wearable devices (as skin temperature $\gg 32^{\circ} \mathrm{C}$, the ambient temperature should be $-28^{\circ} \mathrm{C}$ ).

Despite their low obtained power, energy harvesters could be promising solutions to the replacement battery issue. Moreover, nanotechnology advancement opens up new horizons for reducing battery size while maintaining higher energy output levels.

In the future, energy harvesting should emphasize living subjects and the construction of electronics for self-powered applications, especially for biomedical use. First, we need to make this change to ensure that energy harvester output capacity and efficiency are sustainable enough to fuel electronics. Furthermore, the biocompatibility of equipment is critical. Further integration of advanced engineering concepts with current energy harvesters can provide prospective research guidance. The combination of different energy harvesters with advanced electronics can also pave the way for developing new technologies to diagnose, treat, and prevent diseases.

Acknowledgement: This work was supported and sponsored by Al-Ahliyya Amman University.

Funding Statement: The publication of this paper was partially funded by Al-Ahliyya Amman University.

Conflicts of Interest: The authors declare that they have no conflicts of interest to report regarding the present study.

\section{References}

[1] S. Patel, H. Park, P. Bonato, L. Chan and M. Rodgers, "A review of wearable sensors and systems with application in rehabilitation," Journal of NeuroEngineering and Rehabilitation, vol. 9, no. 1, pp. 21, 2012.

[2] Y. H. Joung, "Development of implantable medical devices: From an engineering perspective," International Neurourology Journal, vol. 17, no. 3, pp. 98, 2013.

[3] A. J. Bandodkar and J. Wang, "Non-invasive wearable electrochemical sensors: A review," Trends in Biotechnology, vol. 32, no. 7, pp. 363-371, 2014.

[4] V. S. Mallela, V. Ilankumaran and N. S. Rao, "Trends in cardiac pacemaker batteries," Indian Pacing and Electrophysiology Journal, vol. 4, pp. 201, 2004.

[5] R. Riemer and A. Shapiro, "Biomechanical energy harvesting from human motion: Theory, state of the art, design guidelines, and future directions," Journal of NeuroEngineering and Rehabilitation, vol. 8, no. 1, pp. 22, 2011.

[6] M. A. Hannan, S. Mutashar, S. A. Samad and A. Hussain, "Energy harvesting for the implantable biomedical devices: issues and challenges," BioMedical Engineering OnLine, vol. 13, no. 1, pp. 79, 2014.

[7] T. Starner, "Human-powered wearable computing," IBM Systems Journal, vol. 35, no. 3.4, pp. 618-629, 1996. 
[8] L. Halámková, J. Halámek, V. Bocharova, A. Szczupak, L. Alfonta et al., "Implanted biofuel cell operating in a living snail," Journal of the American Chemical Society, vol. 134, no. 11, pp. 5040-5043, 2012.

[9] R. Vullers, R. van Schaijk, I. Doms, C. Van Hoof and R. Mertens, "Micropower energy harvesting," Solid-State Electronics, vol. 53, no. 7, pp. 684-693, 2009.

[10] M. N. Adroja, S. B. Mehta and M. P. Shah, "Review of thermoelectricity to improve energy quality," International Journal of Emerging Technologies and Innovative Research JETIR, vol. 2, pp. 847-850, 2015.

[11] M. Hyland, H. Hunter, J. Liu, E. Veety and D. Vashaee, "Wearable thermoelectric generators for human body heat harvesting," Applied Energy, vol. 182, no. 4, pp. 518-524, 2016.

[12] H. Liu, Y. Wang, D. Mei, Y. Shi and Z. Chen, "Design of a wearable thermoelectric generator for harvesting human body energy," in Wearable Sensors and Robots, 1st ed., Singapore: Springer, pp. 55-66, 2017. [Online]. Available: https://link.springer.com/book/10.1007/978-981-10-2404-7.

[13] A. Nozariasbmarz, F. Suarez, J. H. Dycus, M. J. Cabral, J. M. LeBeau et al., "Thermoelectric generators for wearable body heat harvesting: Material and device concurrent optimization," Nano Energy, vol. 67, no. 3, pp. 104265, 2020.

[14] Y. Sargolzaeiaval, V. P. Ramesh, T. V. Neumann, V. Misra, D. Vashaee et al., "Flexible thermoelectric generators for body heat harvesting-enhanced device performance using high thermal conductivity elastomer encapsulation on liquid metal interconnects," Applied Energy, vol. 262, no. 12, pp. 114370, 2020.

[15] Y. Liu, S. Zhang, Y. Zhou, M. A. Buckingham, L. Aldous et al., "Advanced wearable thermocells for body heat harvesting," Advanced Energy Materials, vol. 10, no. 48, pp. 2002539, 2020.

[16] X. Zhao, C. Zhao, Y. Jiang, X. Ji, F. Kong et al., "Flexible cellulose nanofiber/Bi2Te3 composite film for wearable thermoelectric devices," Journal of Power Sources, vol. 479, pp. 229044, 2020.

[17] N. Wen, Z. Fan, S. Yang, Y. Zhao, T. Cong et al., "Highly conductive, ultra-flexible and continuously processable PEDOT: PSS fibers with high thermoelectric properties for wearable energy harvesting," Nano Energy, vol. 78, pp. 105361, 2020.

[18] O. A. Saraereh, A. Alsaraira, I. Khan and B. J. Choi, "A hybrid energy harvesting design for on-body Internet-ofThings (IoT) networks," Sensors, vol. 20, pp. 407, 2020.

[19] M. Zadan, M. H. Malakooti and C. Majidi, "Soft and stretchable thermoelectric generators enabled by liquid metal Elastomer composites," ACS Applied Materials \& Interfaces, vol. 12, no. 15, pp. 17921-17928, 2020.

[20] J. Yuan and R. Zhu, "A fully self-powered wearable monitoring system with systematically optimized flexible thermoelectric generator," Applied Energy, vol. 271, pp. 115250, 2020.

[21] A. Lund, Y. Tian, S. Darabi and C. Müller, "A polymer-based textile thermoelectric generator for wearable energy harvesting," Journal of Power Sources, vol. 480, pp. 228836, 2020.

[22] D. L. Wen, H. T. Deng, X. Liu, G. K. Li, X. R. Zhang et al., "Wearable multi-sensing double-chain thermoelectric generator," Microsystems \& Nanoengineering, vol. 6, no. 1, pp. 1-13, 2020.

[23] S. Li and Q. Zhang, "Ionic gelatin thermoelectric generators," Joule, vol. 4, no. 8, pp. 1628-1629, 2020.

[24] J. Kim, S. Khan, P. Wu, S. Park, H. Park et al., "Self-charging wearables for continuous health monitoring," Nano Energy, vol. 79, pp. 105419, 2021.

[25] T. Sun, B. Zhou, Q. Zheng, L. Wang, W. Jiang et al., "Stretchable fabric generates electric power from woven thermoelectric fibers," Nature Communications, vol. 11, no. 1, pp. 1-10, 2020.

[26] C. Seetawan, A. Seetawan, C. Paiyasen, P. Chaiyapet, S. Thaowankaew et al., "Flexible thermoelectric thin film devices," SNRU Journal of Science and Technology, vol. 12, pp. 155-163, 2020.

[27] X. Zhang, T. T. Li, H. T. Ren, H. K. Peng, J. Qian et al., "Flexible and wearable wristband for harvesting human body heat based on coral-like PEDOT: Tos-coated nanofibrous film," Smart Materials and Structures, vol. 30, no. 1, pp. 015003, 2021.

[28] A. Zurbuchen, A. Pfenniger, A. Stahel, C. T. Stoeck, S. Vandenberghe et al., "Energy harvesting from the beating heart by a mass imbalance oscillation generator," Annals of Biomedical Engineering, vol. 41, no. 1, pp. 131-141, 2013.

[29] M. Amin Karami and D. J. Inman, "Powering pacemakers from heartbeat vibrations using linear and nonlinear energy harvesters," Applied Physics Letters, vol. 100, no. 4, pp. 042901, 2012. 
[30] A. Pfenniger, L. N. Wickramarathna, R. Vogel and V. M. Koch, "Design and realization of an energy harvester using pulsating arterial pressure," Medical Engineering \& Physics, vol. 35, no. 9, pp. 1256-1265, 2013.

[31] G. T. Hwang, H. Park, J. H. Lee, S. Oh, K. I. Park et al., "Self-powered cardiac pacemaker enabled by flexible single crystalline PMN-PT piezoelectric energy harvester," Advanced Materials, vol. 26, no. 28, pp. 4880-4887, 2014.

[32] H. Zhang, X. S. Zhang, X. Cheng, Y. Liu, M. Han et al., "A flexible and implantable piezoelectric generator harvesting energy from the pulsation of ascending aorta: in vitro and in vivo studies," Nano Energy, vol. 12, pp. 296-304, 2015.

[33] B. Lu, Y. Chen, D. Ou, H. Chen, L. Diao et al., "Ultra-flexible piezoelectric devices integrated with heart to harvest the biomechanical energy," Scientific Reports, vol. 5, no. 1, pp. 14, 2015.

[34] M. Ansari and M. A. Karami, "Piezoelectric energy harvesting from heartbeat vibrations for leadless pacemakers," in Journal of Physics: Conference Series. Vol. 660, IOP publishing, pp. 012121, 2015.

[35] A. Zurbuchen, A. Haeberlin, L. Bereuter, A. Pfenniger, S. Bosshard et al., "Endocardial energy harvesting by electromagnetic induction," IEEE Transactions on Biomedical Engineering, vol. 65, no. 2, pp. 424-430, 2018.

[36] L. Dong, X. Han, Z. Xu, A. B. Closson, Y. Liu et al., "Flexible porous piezoelectric cantilever on a pacemaker lead for compact energy harvesting," Advanced Materials Technologies, vol. 4, no. 1, pp. 1800148, 2019.

[37] N. Li, Z. Yi, Y. Ma, F. Xie, Y. Huang et al., "Direct powering a real cardiac pacemaker by natural energy of a heartbeat," ACS Nano, vol. 13, no. 3, pp. 2822-2830, 2019.

[38] L. Dong, A. B. Closson, C. Jin, Y. Nie, A. Cabe et al., "Multifunctional pacemaker lead for cardiac energy harvesting and pressure sensing," Advanced Healthcare Materials, vol. 9, no. 11, pp. 2000053, 2020.

[39] N. Franzina, A. Zurbuchen, A. Zumbrunnen, T. Niederhauser, T. Reichlin et al., "A miniaturized endocardial electromagnetic energy harvester for leadless cardiac pacemakers," PLOS One, vol. 15, no. 9, pp. e0239667, 2020.

[40] Z. Xu, C. Jin, A. Cabe, D. Escobedo, N. Hao et al., "Flexible energy harvester on a pacemaker lead using multibeam piezoelectric composite thin films," ACS Applied Materials \& Interfaces, vol. 12, no. 30, pp. 34170-34179, 2020.

[41] A. Nanda and M. A. Karami, "Energy harvesting from arterial blood pressure for powering embedded micro sensors in human brain," Journal of Applied Physics, vol. 121, no. 12, pp. 124506, 2017.

[42] P. Nwagoum Tuwa and P. Woafo, "Micro-plate piezoelectric energy harvester for pulsating arterial pressure," Journal of Mechanics in Medicine and Biology, vol. 16, pp. 1650073, 2016.

[43] T. W. Secord and M. C. Audi, "A tunable resonance cantilever for cardiac energy harvesting," Cardiovascular Engineering and Technology, vol. 10, no. 2, pp. 380-393, 2019.

[44] M. Angelika-Nikita, N. Hadjigeorgiou, C. Manopoulos and J. Georgiou, "Converting energy captured from blood flow into usable electric power: Design optimisation," arXiv:1809.10929 [physics.med-ph], 2018.

[45] M. G. Abdelmageed, A. M. F. El-Bab and A. Abouelsoud, "Design and simulation of pulsatile blood flow energy harvester for powering medical devices," Microelectronics Journal, vol. 86, pp. 105-113, 2019.

[46] Z. Zhou, L. Weng, T. Tat, A. Libanori, Z. Lin et al., "Smart insole for robust wearable biomechanical energy harvesting in harsh environments," ACS Nano, vol. 14, no. 10, pp. 14126-14133, 2020.

[47] D. W. Lee, D. G. Jeong, J. H. Kim, H. S. Kim, G. Murillo et al., "Polarization-controlled PVDF-based hybrid nanogenerator for an effective vibrational energy harvesting from human foot," Nano Energy, vol. 76, pp. 105066, 2020.

[48] M. Kashfi, P. Fakhri, B. Amini, N. Yavari, B. Rashidi et al., "A novel approach to determining piezoelectric properties of nanogenerators based on PVDF nanofibers using iterative finite element simulation for walking energy harvesting," Journal of Industrial Textiles, vol. 49, pp. 1528083720926493, 2020.

[49] J. Zhao and Z. You, "A shoe-embedded piezoelectric energy harvester for wearable sensors," Sensors, vol. 14, no. 7, pp. 12497-12510, 2014.

[50] I. Choudhry, H. R. Khalid and H. K. Lee, "Flexible piezoelectric transducers for energy harvesting and sensing from human kinematics," ACS Applied Electronic Materials, vol. 2, no. 10, pp. 3346-3357, 2020. 